\title{
Approximate Solution of Nonlinear Integral Equations of the Second Kind by Using Homotopy Perturbation Method
}

\author{
M. M. Hasan* and M. A. Matin \\ Department of Mathematics, Dhaka University, Dhaka-1000, Bangladedsh
}

(Received: 7 February 2017; Accepted 27 March 2017)

\begin{abstract}
In this paper, we apply Homotopy perturbation method (HPM) for obtaining approximate solution of nonlinear Fredholm integral equation of the second kind. Finally, some numerical examples are provided, and the obtained numerical approximations are compared with the corresponding exact solution.
\end{abstract}

Keywords: Nonlinear Fredholm integral equation, Homotopy perturbation method, approximate solutions, Origin Pro 8 and MATHEMATICAv9 softwares.

\section{Introduction}

In recent years, integral equations have involved the attention of many scientists and researchers due to their wide range of applications in science and technology. Integral equations arise in the potential theory more than any other field. Integral equations arise also in diffraction problems, conformal mapping, water waves, scattering in quantum mechanics, and population growth model. The electrostatic, electromagnetic scatering problems and propagation of acoustical and elastically waves are scientific fields where integral equations appear ${ }^{5}$. The Fredholm integral equation is of widespread use in many realms of engineering and applied mathematics. Consider the general form non-linear Fredholm integral equation of the second kind

$$
\begin{gathered}
y(x)=f(x)+\lambda \int_{a}^{b} K(x, t) F(y(t)) d t, \\
a \leq x \leq b
\end{gathered}
$$

where $y(x)$ is the unknown solution, $a$ and $b$ are real constants. The kernel $K(x, t)$ and $f(x)$ are known smooth functions on $R^{2}$ and $R$ respectively. The parameter $\lambda$ is a real (or complex) known as the eigenvalue when $\lambda$ is a real parameter, and $F$ is a nonlinear function of $y$.

\section{Homotopy Perturbation Method}

Suppose the following nonlinear Fredholm integral equation of the second kind of the form

$$
\begin{gathered}
y(x)=f(x)+\int_{0}^{1} K(x, t) F(y(t)) d t \\
0 \leq x \leq 1
\end{gathered}
$$

We assume $F(y(t))$ is a nonlinear function of $y(x)$. That means that the nonlinear Fredholm integral equation (1) contains the nonlinear function presented by $F(y(t))$.

For solving equation (1) by Homotopy perturbation method $(\mathrm{HPM})^{1,2,4}$, we consider equation (1) as

$$
\begin{gathered}
L(y)=y(x)-f(x) \\
-\int_{0}^{1} K(x, t) F(y(t)) d t=0
\end{gathered}
$$

We define the homotopy $H(y, p)$ by

$$
\left.\begin{array}{rl}
H(y, 0) & =N(y) \\
H(y, 1) & =L(y)
\end{array}\right\}
$$

where $N(y)$ is an integral operator with known solution $y_{0}$. We next construct a convex homotopy of the form

$$
H(y, p)=(1-p) N(y)+p L(y)=0
$$

and continuously trace an implicitly defined curve from a starting point $H\left(y_{0}, 0\right)$ to a solution function $H(y, 1)$. The embedding parameter $p$ monotonically increases from zero to unit as the trivial problem $L(y)=0$. The embedding parameter $p \in(0,1]$ can be considered as an expanding parameter. The HPM uses the homotopy parameter $p$ as an expanding parameter to obtain

$$
\begin{gathered}
y=\sum_{i=0}^{\infty} p^{i} y_{i}(x)=y_{0}+p^{1} y_{1}+p^{2} y_{2} \\
+p^{3} y_{3}+\cdots
\end{gathered}
$$

When $p \rightarrow 1$, (5a) corresponding to (4) become the approximate solution of (2) as follows

$$
\begin{aligned}
y & =\lim _{p \rightarrow 1} \sum_{i=0}^{\infty} p^{i} y_{i}(x) \\
& =y_{0}+y_{1}+y_{2}+\cdots
\end{aligned}
$$

The series in (5b) converges in most cases, and the rate of convergence depend on $L(y)$.

Consider

$$
N(y)=y(x)-f(x)
$$

The nonlinear term $F(y(t))$ can be expressed in $\mathrm{He}$ polynomials ${ }^{3}$ as

$$
\begin{aligned}
F(y)=\sum_{k=0}^{\infty} p^{k} H_{k}\left(y_{0}, y_{1}, y_{2}, \ldots, y_{k}\right) & \\
=H_{0}\left(y_{0}\right) & +p^{1} H_{1}\left(y_{0}, y_{1}\right)+ \\
& \ldots+p^{k} H_{k}\left(y_{0}, y_{1}, \ldots \ldots ., y_{k}\right)
\end{aligned}
$$

where

$$
\begin{gathered}
H_{k}\left(y_{0}, \ldots, y_{k}\right)=\frac{1}{k !} \frac{d^{k}}{d p^{k}}\left[F\left(\sum_{i=0}^{k} p^{i} y_{i}\right)\right], \\
k=0,1,2 \ldots . .
\end{gathered}
$$

\footnotetext{
* Author for correspondence. e-mail: mohedul.math@gmail.com
} 
Using (5a), (6), and (7) into (4), we have

$$
\begin{gathered}
(1-p)\left(\left(y_{0}+p^{1} y_{1}+\cdots\right)-f(x)\right) \\
+p\left(\left(y_{0}+p^{1} y_{1}+p^{2} y_{2}+\cdots\right)-f(x)\right. \\
\left.-\int_{0}^{1} K(x, t) \sum_{j=0}^{\infty}\left(p^{j} H_{j}\right) d t\right)=0 \\
\Rightarrow\left(y_{0}+p^{1} y_{1}+p^{2} y_{2}+\cdots\right)-f(x) \\
-p \int_{0}^{1} K(x, t) \sum_{j=0}^{\infty}\left(p^{j} H_{j}\right) d t=0
\end{gathered}
$$

Equating the term with identical power of $p$ in equation (9),

$p^{0}: y_{0}(x)-f(x)=0 \Rightarrow y_{0}(x)=f(x)$

$p^{1}: y_{1}(x)-\int_{0}^{1} K(x, t) H_{0}(t) d t=0$

$\Rightarrow y_{1}(x)=\int_{0}^{1} K(x, t) H_{0}(t) d t$

and so on.

And in general form we have

$$
\begin{gathered}
y_{0}(x)=f(x) \\
y_{k+1}(x)=\int_{0}^{1} K(x, t) H_{k}(t) d t \\
k=0,1,2, \ldots
\end{gathered}
$$

Using the recursive scheme (10), the $n$-term approximation series solution can be obtained as follows:

$$
\varphi_{n}(x)=\sum_{j=0}^{n} y_{j}(x)
$$

\section{Numerical Implementations}

In this section, we will apply the Homotopy perturbation method to compute a numerical solution for non-linear integral equation of the Fredholm type. Then we will compare between the results which we obtain by the numerical solution technique and the results of the exact solution. To illustrate this, we consider the following example:

\section{Example 1}

Consider the following nonlinear Fredholm integral equation of the second kind

$$
y(x)=\frac{7}{8} x+\frac{1}{2} \int_{0}^{1} x t y^{2}(t) d t
$$

where the exact solution of the equation is $y(x)=x$. In the following, we will compute the polynomials for the nonlinear terms $y^{2}(t)$ that arises in nonlinear integral equation.

For $k=0$, equation (4) becomes

$$
\begin{aligned}
H_{0}= & \frac{1}{0 !} \frac{d^{0}}{d p^{0}}\left[F\left(\sum_{i=0}^{\infty} p^{i} y_{i}\right)\right]_{p=0} \\
= & {\left[F\left(\sum_{i=0}^{\infty} p^{i} y_{i}\right)\right]_{p=0} } \\
& =\left[F\left(p^{0} y_{0}+p^{1} y_{1}+p^{2} y_{2}+\cdots\right)\right]_{p=0}
\end{aligned}
$$

The polynomials for $F(y)=y^{2}$ are given by

$$
=\left.\left(y_{0}+p y_{1}+p^{2} y_{2}+\cdots\right)^{2}\right|_{p=0}
$$

$\therefore H_{0}=y_{0}^{2}$

By using the MATHEMATICA software, the next few terms, we have

$H_{1}=2 y_{0} y_{1}$

$H_{2}=2 y_{0} y_{2}+y_{1}^{2}$

and so on.

Applying the technique as stated above in equation (10), we have

$p^{0}: y_{0}(x)=\frac{7}{8} x$

$p^{1}: y_{1}(x)=\frac{1}{2} \int_{0}^{1} x t H_{0}(t) d t=\frac{7^{2}}{8^{3}} x$

In a similar manner, to obtain the iteration at the ninth step. Therefore we can write

$$
\begin{aligned}
y(x)= & \left(\frac{7}{8}+\frac{7^{2}}{8^{3}}+\frac{7^{3}}{4 \times 8^{4}}+\frac{5 \times 7^{4}}{8^{7}}+\frac{7^{6}}{4 \times 8^{8}}\right. \\
& +\frac{3.7^{7}}{4 \times 8^{10}}+\frac{1811 \times 7^{6}}{2 \times 8^{14}}+\frac{5 \times 283 \times 7^{8}}{4 \times 8^{15}} \\
& \left.+\frac{5 \times 3673 \times 7^{9}}{2 \times 8^{18}}+\frac{5 \times 798101 \times 7^{10}}{2 \times 8^{22}}\right) x
\end{aligned}
$$

$\approx 0.999947 x$

The table under shows the approximate solutions obtained by applying the Homotopy perturbation method giving to the value of $x$, which is in the interval $[0,1]$.

Table 1. Numerical and exact solutions to the integral equation (12)

\begin{tabular}{cccc}
\hline $\begin{array}{c}\text { Nodes } \\
(\mathrm{x})\end{array}$ & $\begin{array}{c}\text { Exact } \\
\text { solutions }\end{array}$ & $\begin{array}{c}\text { Approximate } \\
\text { solutions }\end{array}$ & $\begin{array}{c}\text { Absolute } \\
\text { Error }\end{array}$ \\
\hline 0 & 0 & 0 & 0 \\
0.10 & 0.100000 & 0.0999947 & 0.0000053 \\
0.20 & 0.200000 & 0.1999890 & 0.0000110 \\
0.30 & 0.300000 & 0.2999840 & 0.0000160 \\
0.40 & 0.400000 & 0.3999790 & 0.0000210 \\
0.50 & 0.500000 & 0.4999740 & 0.0000260 \\
0.60 & 0.600000 & 0.5999680 & 0.0000320 \\
0.70 & 0.700000 & 0.6999630 & 0.0000370 \\
0.80 & 0.800000 & 0.7999580 & 0.0000420 \\
0.90 & 0.900000 & 0.8999520 & 0.0000480 \\
1.0 & 1.000000 & 0.9999470 & 0.0000530 \\
\hline
\end{tabular}




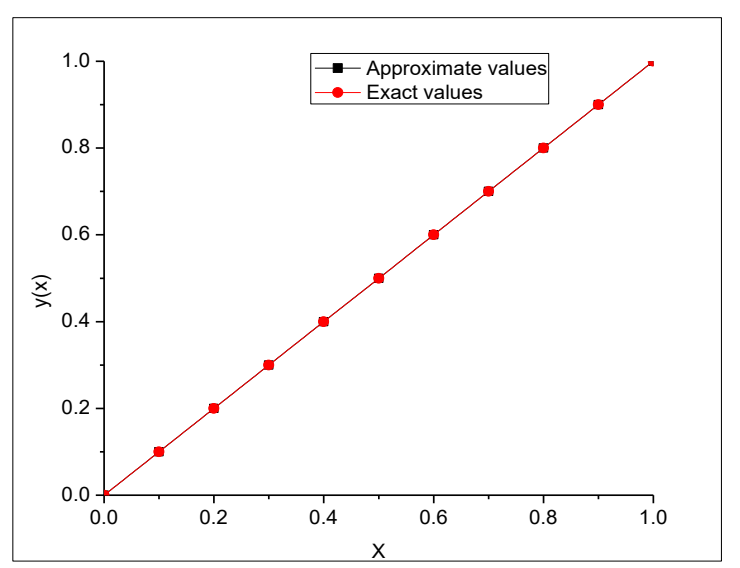

Fig. 1. Numerical and exact solutions to the integral equation (12)

\section{Example 2}

Consider the following nonlinear Fredholm integral equation of the second kind

$$
y(x)=3+0.6625 x+\frac{x}{20} \int_{0}^{1} t y^{2}(t) d t
$$

Applying above procedure, we have

$p^{0}: y_{0}(x)=3+0.6625 x$

$p^{1}: y_{1}(x)=\frac{x}{20} \int_{0}^{1} t y_{0}{ }^{2}(t) d t=0.296736 x$

In a similar manner, to obtain the iteration at the ninth step. Therefore we can write

$$
\begin{gathered}
y(x)=3+(0.6625+0.296736 \\
+0.0345883+0.0356889 \\
+0.00441658+0.000794517 \\
+0.000156235+0.0000438455 \\
+0.0000069959+0.000002199) x \\
\approx 3+1.03493 x
\end{gathered}
$$

The table below shows the approximate solutions obtained by applying the Homotopy perturbation method according to the value of $x$, which is in the interval $[0,1]$.

Table 2. Numerical and exact solutions to the integral equation (13)

\begin{tabular}{cccc}
\hline $\begin{array}{c}\text { Nodes } \\
(\mathrm{x})\end{array}$ & $\begin{array}{c}\text { Exact } \\
\text { solutions }\end{array}$ & $\begin{array}{c}\text { Approximate } \\
\text { solutions }\end{array}$ & $\begin{array}{c}\text { Absolute } \\
\text { Error }\end{array}$ \\
\hline 0 & 3 & 3 & 0 \\
0.10 & 3.100000 & 3.10349 & 0.00349 \\
0.20 & 3.200000 & 3.20699 & 0.00699 \\
0.30 & 3.300000 & 3.31048 & 0.01048 \\
0.40 & 3.400000 & 3.41397 & 0.01397 \\
0.50 & 3.500000 & 3.51747 & 0.01747 \\
0.60 & 3.600000 & 3.62096 & 0.02096 \\
0.70 & 3.700000 & 3.72445 & 0.02445 \\
0.80 & 3.800000 & 3.82794 & 0.02794 \\
0.90 & 3.900000 & 3.93144 & 0.03144 \\
1.0 & 4.000000 & 4.03493 & 0.03493 \\
\hline
\end{tabular}

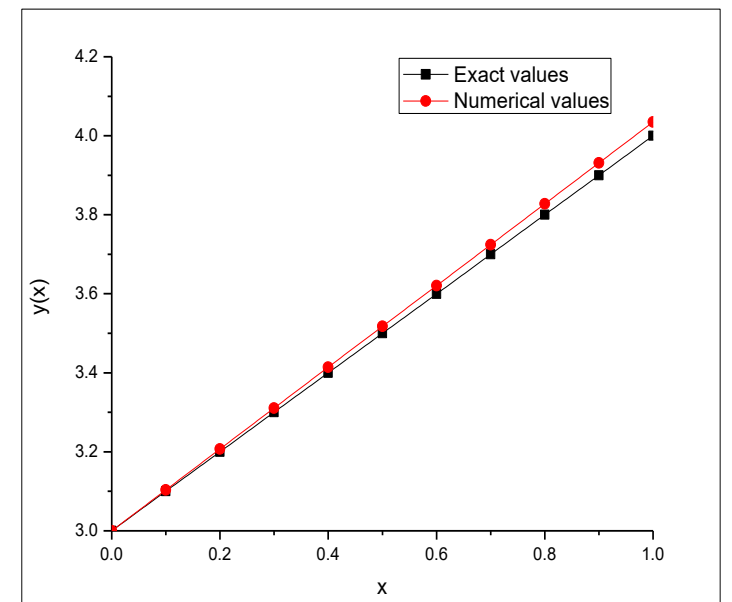

Fig. 2. Numerical and exact solutions to the integral equation (13)

\section{Example 3}

Consider the following nonlinear Fredholm integral equation

$$
\begin{aligned}
& y(x)=\sin (\pi x)+\frac{1}{5} \int_{0}^{1} \cos (\pi x) \sin (\pi t)(y(t))^{3} d t \\
& x \in[0,1] \\
& \text { The exact solution of the equation }(10) \text { is } y(x)= \\
& \sin (\pi x)+\frac{1}{3}(20-\sqrt{391}) \cos (\pi x) .
\end{aligned}
$$

In the following, we will calculate the polynomials for the nonlinear terms $y^{3}(t)$ that arises in nonlinear integral equation.

For $k=0$, equation (4) becomes

$$
\begin{gathered}
H_{0}=\frac{1}{0 !} \frac{d^{0}}{d p^{0}}\left[F\left(\sum_{i=0}^{\infty} p^{i} y_{i}\right)\right]_{p=0} \\
=\left[F\left(\sum_{i=0}^{\infty} p^{i} y_{i}\right)\right]_{p=0} \\
=\left[F\left(p^{0} y_{0}+p^{1} y_{1}+p^{2} y_{2}+\cdots\right)\right]_{p=0}
\end{gathered}
$$

Now the polynomials for $F(y)=y^{3}$ are given by $=\left.\left(y_{0}+p y_{1}+p^{2} y_{2}+\cdots\right)^{3}\right|_{p=0}$

$\therefore H_{0}=y_{0}{ }^{3}$

By using the MATHEMATICA v9 software, the next few terms, we have

$H_{1}=3 y_{0}^{2} y_{1}$

$H_{2}=3\left(y_{0} y_{1}^{2}+y_{0}^{2} y_{2}\right)$

$H_{3}=y_{1}{ }^{3}+6 y_{0} y_{1} y_{2}+3 y_{0}{ }^{2} y_{3}$

$H_{4}=3\left(y_{1}^{2} y_{2}+y_{0} y_{2}^{2}+y_{0}^{2} y_{4}\right)+6 y_{0} y_{1} y_{3}$

$H_{5}=3\left(y_{1} y_{2}{ }^{2}+y_{1}{ }^{2} y_{3}+y_{0}{ }^{2} y_{5}\right)+6\left(y_{0} y_{2} y_{3}+y_{0} y_{1} y_{4}\right)$

$H_{6}=y_{2}^{3}+6\left(y_{1} y_{2} y_{3}+y_{0} y_{2} y_{4}+y_{0} y_{1} y_{5}\right)$

$+3\left(y_{0} y_{3}{ }^{2}+y_{1}{ }^{2} y_{4}+y_{0}{ }^{2} y_{6}\right)$
$+y_{7}$

$H_{7}=3\left(y_{2}^{2} y_{3}+y_{1} y_{3}^{2}+y_{1}^{2} y_{5}+y_{0}^{2} y_{7}\right)+6\left(y_{1} y_{2} y_{4}\right.$

and so on.

$\left.+y_{0} y_{3} y_{4}+y_{0} y_{2} y_{5}+y_{0} y_{1} y_{6}\right)$

Applying the procedure as stated above in equation (10), we have

$p^{0}: y_{0}(x)=\sin (\pi x)$ 


$$
\begin{aligned}
p^{1}: y_{1}(x) & =\frac{\cos (\pi x)}{5} \int_{0}^{1} \sin (\pi t) H_{0}(t) d t \\
& =\frac{3}{40} \cos (\pi x)
\end{aligned}
$$

In a similar manner, to obtain the iteration at the ninth step. Therefore we can write

$$
\begin{gathered}
y(x)=\sin (\pi x)+\left(\frac{3}{40}+0+\frac{27}{64000}+0\right. \\
+\frac{243}{51200000}+0+\frac{2187}{32768000000}+
\end{gathered}
$$

$$
\begin{gathered}
\left.0+\frac{137781}{131072000000000}\right) \cos (\pi x) \\
=\sin (\pi x)+\frac{9886326965781}{131072000000000} \cos (\pi x)
\end{gathered}
$$

The table under shows the approximate solutions obtained by applying the Homotopy perturbation method according to the value of $x$ in $[0,1]$.

Table 3. Numerical and exact solutions to the integral equation (14)

\begin{tabular}{cccc}
\hline Nodes & Exact values & Approximate values & Absolute error \\
\hline 0.00 & 0.07542668890493687 & 0.07542668888687896 & $1.8057902 \times 10^{-11}$ \\
0.05 & 0.23093252624133365 & 0.23093252622349808 & $1.7835566 \times 10^{-11}$ \\
0.10 & 0.38075203836055493 & 0.3807520383433809 & $1.7174039 \times 10^{-11}$ \\
0.15 & 0.5211961716517719 & 0.5211961716356822 & $1.6089685 \times 10^{-11}$ \\
0.20 & 0.6488067254459994 & 0.6488067254313902 & $1.4609202 \times 10^{-11}$ \\
0.25 & 0.7604415043936764 & 0.7604415043809076 & $1.2768786 \times 10^{-11}$ \\
0.30 & 0.8533516897425216 & 0.8533516897319074 & $1.0614176 \times 10^{-11}$ \\
0.35 & 0.9252495243780194 & 0.9252495243698213 & $8.198108 \times 10^{-12}$ \\
0.40 & 0.9743646449962113 & 0.9743646449906311 & $5.580202 \times 10^{-12}$ \\
0.45 & 0.9994876743237375 & 0.9994876743209126 & $2.824962 \times 10^{-12}$ \\
0.50 & 1 & & 1 \\
0.55 & 0.9758890068665379 & 0.9758890068693629 & 0 \\
0.60 & 0.9277483875940958 & 0.927748387599676 & $2.824962 \times 10^{-12}$ \\
0.65 & 0.8567635239987162 & 0.8567635240069144 & $8.198108 \times 10^{-12}$ \\
0.70 & 0.7646822990073733 & 0.7646822990179875 & $1.0614176 \times 10^{-11}$ \\
0.75 & 0.6537720579794186 & 0.6537720579921874 & $1.2768786 \times 10^{-11}$ \\
0.80 & 0.526763779138947 & 0.5267637791535562 & $1.4609202 \times 10^{-11}$ \\
0.85 & 0.38678482782732176 & 0.38678482784341145 & $1.6089685 \times 10^{-11}$ \\
0.90 & 0.23728195038933994 & 0.237281950406514 & $1.7174067 \times 10^{-11}$ \\
0.95 & 0.08193640383912822 & 0.08193640385696378 & $1.7835566 \times 10^{-11}$ \\
1.00 & -0.07542668890493687 & -0.07542668888687896 & $1.8057902 \times 10^{-11}$ \\
\hline
\end{tabular}

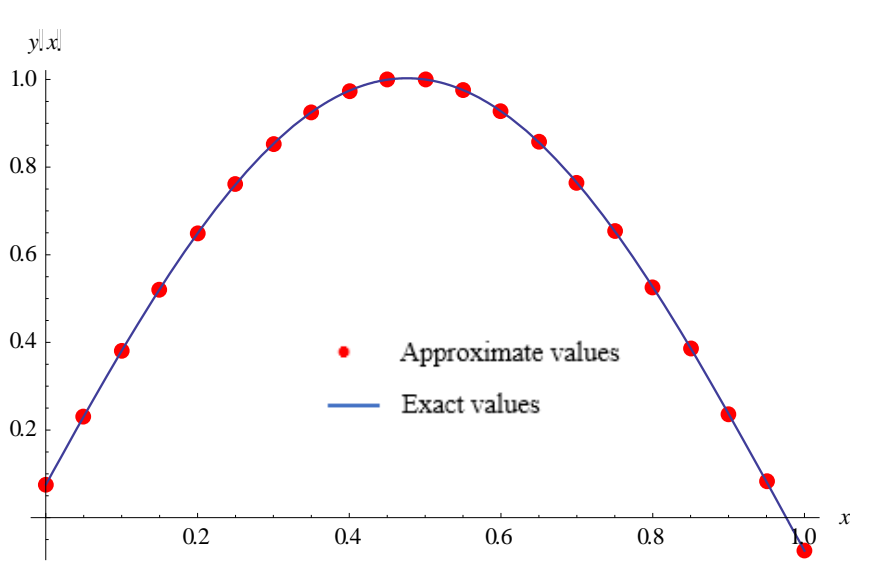

Fig. 3. Numerical and exact solutions to the integral equation (14)

\section{Conclusion}

This paper presents a method to find the solution of a nonlinear Fredholm integral equation by Homotopy perturbation method (HPM). The approximate solutions obtained by the HPM are compared with exact solutions. It can be concluded that the HPM is effective and reliable and accuracy of the numerical results indicates that the proposed method is well suited for the solution of such type problems. These results corroborate the results obtained in 6 done by Newton-Kantorovich method and Adomian decomposition method. 


\section{References}

1. Abbasbandy S., 2011. Numerical solutions of the integral equations: homotopy perturbation method and Adomian's decomposition method, Applied Mathematics and Computation, 173(1), 493-500.

2. Gajni D. D., G. A. Afrouzi, H. Hosseinzadeh, and R. A.Talarposhti, 2007.Application of homotopy perturbation method to the second kind of nonlinear integral equations, Physics Letters A, 371(1-2), 20-22.

3. Ghorbani A., 2009. Beyond Adomian polynomials: He polynomials, Chaos, Solitons \& Fractals, 39(3), 1486-1492.

4. Hasan M. M., M. A. Matin, 2017. Numerical Solution of Nonlinear Fredholm Integral Equations by using NKM and ADM, Dhaka Univ. J. Sci. 65(1), 61-66.

5. Javidi M. and A. Golbabai, 2009. Modified homotopy perturbation method for solving nonlinear Fredholm integral equations, Chaos, Solitons and Fractals, 40(3), 1408-1412.-66.

6. Wazwaz A. M., 2015. A First Course in Integral Equations, World Scientific. 
